\title{
The Higher Order Riesz Transform and BMO Type Space Associated with Schrödinger Operators on Stratified Lie Groups
}

\author{
Yu Liu ${ }^{1}$ and Jianfeng Dong ${ }^{2}$ \\ ${ }^{1}$ School of Mathematics and Physics, University of Science and Technology Beijing, Beijing 100083, China \\ ${ }^{2}$ Department of Mathematics, Shanghai University, Shanghai 200444, China \\ Correspondence should be addressed to Yu Liu; liuyu75@pku.org.cn
}

Received 1 October 2013; Accepted 7 November 2013

Academic Editor: Yoshihiro Sawano

Copyright (c) 2013 Y. Liu and J. Dong. This is an open access article distributed under the Creative Commons Attribution License, which permits unrestricted use, distribution, and reproduction in any medium, provided the original work is properly cited.

\begin{abstract}
Assume that $G$ is a stratified Lie group and $Q$ is the homogeneous dimension of $G$. Let $-\Delta$ be the sub-Laplacian on $G$ and $W \not \equiv 0$ a nonnegative potential belonging to certain reverse Hölder class $B_{s}$ for $s \geq Q / 2$. Let $L=-\Delta+W$ be a Schrödinger operator on the stratified Lie group $G$. In this paper, we prove the boundedness of some integral operators related to $L$, such as $L^{-1} \nabla^{2}, L^{-1} W$, and $L^{-1}(-\Delta)$ on the space $B M O_{L}(G)$.
\end{abstract}

\section{Introduction}

In recent years, some problems related to Schrödinger operators on the Euclidean space $\mathbb{R}^{n}$ with nonnegative potentials have been investigated by a number of scholars (cf. [112 ], etc.). Later, more scholars want to generalize the above results related to Schrödinger operators to a more general setting, such as Heisenberg group, nilpotent Lie groups, and spaces of homogeneous type (cf. [13-24], etc.). The auxiliary function plays an important role in the Harmonic analysis problems related to Schrödinger operators. Recently, Yang et al. introduced the admissible function. It is known that the auxiliary function is a special case of the admissible function. Accordingly, they investigated function spaces, such as $B M O$, $B L O$, and Hardy space, related to the admissible function in $[22,24]$. Among the above problems, Riesz transforms and higher order Riesz transforms related to Schrödinger operators are one of hottest issues. Their $L^{p}$ boundedness has been obtained by Shen [13] and $\mathrm{Li}$ [4] in the different settings. Dziubański and Zienkiewicz proved that Riesz transforms related to Schrödinger operators are bounded from Hardy spaces associated with Schrödinger operators into $L^{1}$ in [1]. Endpoint boundedness of Riesz transforms related to Schrödinger operators had been investigated in $[11,25]$. Dong and Liu established the BMO spaces associated with Schrödinger operators for the Riesz transform related to Schrödinger operators in [26]. Lin et al. obtained the corresponding results on the Heisenberg group in $[14,15]$. Just now, Dong and Liu established the BMO estimates for the higher order Riesz transform in [27]. The aim of this paper is to obtain the BMO estimates for the higher order transform on stratified Lie groups.

Firstly, we recall some basic facts of stratified Lie groups (cf. [28]). A Lie group $G$ is called stratified if it is nilpotent, connected, and simple connected, and its Lie algebra $\mathfrak{g}$ admits a vector space decomposition $\mathfrak{g}=V_{1} \oplus \cdots \oplus V_{m}$ such that $\left[V_{1}, V_{k}\right]=V_{k+1}$ for $1 \leq k<m$ and $\left[V_{1}, V_{m}\right]=0$. If $G$ is stratified, its Lie algebra admits a family of dilations, namely,

$$
\begin{aligned}
\delta_{r}\left(X_{1}\right. & \left.+X_{2}+\cdots+X_{m}\right) \\
= & r X_{1}+r^{2} X^{2}+\cdots+r^{m} X^{m} \\
& \times\left(X_{j} \in V_{j}, j \in\{1, \ldots, m\}\right) .
\end{aligned}
$$

Assume that $G$ is a Lie group with underlying manifold $\mathbb{R}^{n}$ for some positive integer $n$. $G$ inherits dilations from $\mathfrak{g}$ : if $x \in G$ and $r>0$, we write

$$
\delta_{r} x=\left(r^{d_{1}} x_{1}, \ldots, r^{d_{n}} x_{n}\right)
$$


where $1 \leq d_{1} \leq \cdots \leq d_{n}$. The map $x \rightarrow \delta_{r} x$ is an automorphism of $G$. The left (or right) Haar measure on $G$ is simply $d x_{1} \cdots d x_{n}$, which is the Lebesgue measure on $\mathfrak{g}$. For any measurable set $E \subseteq G$, denote by $|E|$ the measure of $E$. The inverse of any $x \in G$ is simply $x^{-1}=-x$. The group law has the following form:

$$
x y=\left(p_{1}(x, y), \ldots, p_{n}(x, y)\right)
$$

for some polynomials $p_{1}, \ldots, p_{n}$ in $x_{1}, \ldots, x_{n}, y_{1}, \ldots, y_{n}$.

The number $Q=\sum_{j=1}^{m} j\left(\operatorname{dim} V_{j}\right)$ is called the homogeneous dimension of $G$. We fix a homogeneous norm function $|\cdot|$ on $G$, which is smooth away from $e$, where $e$ is the unit element of $G$. Thus, $\left|\delta_{r} x\right|=r|x|$ for all $x \in G, r>0,\left|x^{-1}\right|=$ $|x|$ for all $x \in G$, and $|x|>0$ if $x \neq 0$. The homogeneous norm induces a quasi-metric $d$ which is defined by $d(x, y):=$ $\left|x^{-1} y\right|$. In particular, $d(e, x)=|x|$ and $d(x, y)=d\left(e, x^{-1} y\right)$. The ball of radius $r$ centered at $x$ is written by

$$
B(x, r)=\{y \in G \mid d(x, y)<r\} .
$$

The measure of $B(x, r)$ is

$$
|B(x, r)|=b r^{Q},
$$

where $b$ is a constant. In particular set $V(r)=|B(x, r)|$ for $r>0$ and $x \in G$.

Let $X=\left\{X_{1}, \ldots, X_{l}\right\}$ be a basis for $V_{1}$ (viewed as leftinvariant vector fields on $G$ ). Following [29], one can define a left invariant metric $d_{c}$ associated with $X$ which is called the Carnot-Caratheodory metric: let $x, y \in G$, and for every $\delta>0$ define

$$
\begin{aligned}
& A(\delta) \\
& =\{\gamma:[0, \delta] \longrightarrow G \mid \gamma(0)=x, \gamma(\delta)=y, \\
& \left.\gamma^{\prime}(s)=\sum_{i=1}^{l} a_{i}(s) X_{i}(\gamma(s)) \text { a.e. with } \sum_{i=1}^{l}\left|a_{i}\right| \leq 1\right\} .
\end{aligned}
$$

Let us define

$$
d_{c}(x, y)=\inf _{A(\delta) \text { not empty }}\{\delta\}
$$

The Carnot-Caratheodory metric $d_{c}$ is equivalent to the quasi-metric $d$. From the results of Nagel et al. in [29], we deduce that there exists a constant $a=a(G)>1$ such that, for any $g, h \in G$,

$$
a^{-1} d_{c}(x, y) \leq d(x, y) \leq a d_{c}(x, y)
$$

It follows from [28] that $X_{j}, j=1,2, \ldots, l$, are skew adjoint; that is, $X_{j}^{*}=-X_{j}$. Let $\Delta=-\sum_{i=1}^{l} X_{i}^{2}$ be the subLaplacian on $G$. This operator (which is hypoelliptic by Hörmander's theorem in [30]) plays the same fundamental role on $G$ as the ordinary does on $\mathbb{R}^{n}$. The gradient operator $\nabla$ is denoted by $\nabla=\left(X_{1}, \ldots, X_{l}\right)$.
Definition 1. A nonnegative locally $L^{s}$ integrable function $W$ on $G$ is said to belong to the reverse Hölder class $B_{s}(1<$ $s<\infty)$ if there exists $C>0$ such that the reverse Hölder inequality

$$
\left(\frac{1}{|B(x, r)|} \int_{B(x, r)} W(y)^{s} d y\right)^{1 / s} \leq \frac{C}{|B(x, r)|} \int_{B(x, r)} W(y) d y
$$

holds for every ball $B(x, r)$ in $G$.

Moreover, a locally bounded nonnegative function $W \in$ $B_{\infty}$ if there exists a positive constant $C$ such that

$$
\|W\|_{L^{\infty}(B(x, r))} \leq \frac{C}{|B(x, r)|} \int_{B(x, r)} W(y) d y
$$

holds for every ball $B(x, r)$ in $G$.

Furthermore, it is easy to see that $B_{\infty} \subseteq B_{s}$ for any $1<s<$ $\infty$.

Let $L=-\Delta+W$ be a Schrödinger operator on the stratified Lie group $G$, where $W \not \equiv 0$ is a nonnegative potential belonging to the reverse Hölder class $B_{s}$ for some $s \geq Q / 2$. Denote by $\mathscr{R}^{L}=\nabla^{2} L^{-1}$ the higher order Riesz transform. Accordingly, denote by $\widetilde{\mathscr{R}}^{L}=L^{-1} \nabla^{2}$ its dual operator.

It follows from [13] that the integral operators $W L^{-1}$ and $(-\Delta) L^{-1}$ are bounded on $L^{p}(G)$ for $1 \leq p \leq s$ and $\mathscr{R}^{L}$ is bounded on $L^{p}(G)$ for $1<p \leq s$. Lin et al. introduced the Hardy type space $H_{L}^{1}(G)$ related to the Schrödinger operator $L$ on the Heisenberg group $G$ in [14]. The dual space of $H_{L}^{1}(G)$ is the $B M O$ type space $B M O_{L}(G)$ investigated by Lin and Liu in $[15] . H_{L}^{1}(G)$ and $B M O_{L}(G)$ were also introduced as applications of results in $[11,22]$.

Next, we recall the definition of $H_{L}^{1}(G)$ and $B M O_{L}(G)$. Since $W \geq 0$ and $W \in L_{\text {loc }}^{s}(G)$, the Schrödinger operator $L=-\Delta+W$ generates a $\left(C_{0}\right)$ semigroup $\left\{T_{t}^{L}\right\}_{t>0}=\left\{e^{-t L}\right\}_{t>0}$. The maximal function with respect to the semigroup $\left\{T_{t}^{L}\right\}_{t>0}$ is given by

$$
M^{L} f(x)=\sup _{t>0}\left|T_{t}^{L} f(x)\right|
$$

The Hardy space $H_{L}^{1}(G)$ associated with the Schrödinger operator $L$ is defined as follows in terms of the maximal function mentioned above.

Definition 2. A function $f \in L^{1}(G)$ is said to be in $H_{L}^{1}(G)$ if the semigroup maximal function $M^{L} f$ belongs to $L^{1}(G)$. The norm of such a function is defined by

$$
\|f\|_{H_{L}^{1}}=\left\|M^{L} f\right\|_{L^{1}}
$$

Assume $W \in B_{s}$ for $s>Q / 2$. The auxiliary function $m(x$, $W)$ is defined by

$$
\begin{array}{r}
\rho(x)=\frac{1}{m(x, W)} \doteq \sup _{r>0}\left\{r: \frac{1}{r^{\mathrm{Q}-2}} \int_{B(x, r)} W(y) d y \leq 1\right\}, \\
x \in G .
\end{array}
$$


It follows from Lemma 9 in Section 2 that $0<m(x, W)<\infty$ for any $x \in G$.

The dual space of $H_{L}^{1}(G)$ is the $B M O$ type space $B M O_{L}(G)$ (cf. [22]). Let $f$ be a locally integrable function on $G$ and $B=$ $B(x, r)$ be a ball. Set

$$
\begin{gathered}
f_{B}=\frac{1}{|B(x, r)|} \int_{B(x, r)} f(y) d y, \\
f(B, W)=\left\{\begin{array}{cc}
f_{B}, & \text { if } r<\rho(x), \\
0, & \text { if } r \geq \rho(x) .
\end{array}\right.
\end{gathered}
$$

Definition 3. Let $f$ be a locally integrable function on $G$. One says $f \in B M O_{L}(G)$ if

$$
\|f\|_{B M O_{L}}=\sup _{B} \frac{1}{|B|} \int_{B}|f(y)-f(B, W)| d y<\infty .
$$

It is clear that $L^{\infty}(G) \subset B M O_{L}(G) \subset B M O(G)$ and $\|f\|_{B M O} \leq 2\|f\|_{B M O_{L}}$. Some remarks are given as follows.

Remark 4. Let $1 \leq p<\infty$. If $f \in B M O_{L}(G)$, then there exists a positive constant $C$ :

$$
\sup _{B}\left(\frac{1}{|B|} \int_{B}|f(y)-f(B, W)|^{p} d y\right)^{1 / p} \leq C\|f\|_{B M O_{L}} .
$$

The above inequality can be easily deduced by Lemma 3.1 in [11].

Similar to Remark 1 in [15], we conclude that a function $f \in B M O_{L}(G)$ if and only if there exist some suitable constants $C_{B}$ and $C$ depending on $B=B(x, r)$ and satisfying $C_{B}=0$ whenever $r \geq \rho(x)$ such that

$$
\begin{gathered}
\sup _{B}\left(\frac{1}{|B|} \int_{B}\left|f(y)-C_{B}\right|^{p} d y\right)^{1 / p}<\infty, \\
\|f\|_{B M O_{L}} \leq C \sup _{B}\left(\frac{1}{|B|} \int_{B}\left|f(y)-C_{B}\right|^{p} d y\right)^{1 / p} .
\end{gathered}
$$

Our main results are given as follows.

Theorem 5. Suppose $W \in B_{s}$ for some $s \geq Q / 2$. Then the operators $L^{-1} W$ and $L^{-1}(-\Delta)$ are bounded on the space $B M O_{L}(G)$.

Theorem 6. Suppose $W \in B_{s}$ for some $s \geq Q,|\nabla W| \in B_{s_{1}}$ for some $s_{1} \geq Q / 2$, and $\rho(x) \lesssim \rho_{1}(x)=1 / m(x,|\nabla W|)$ and $\rho_{1}(x) \leq M$ for some positive constant $M$. Then operator $L^{-1} \nabla^{2}$ is bounded on the space $B M O_{L}(G)$.

It shoud be noted that because the left invariant vector fields in $V_{1} \subseteq \mathfrak{g}$ are skew-adjoint and they interact with convolution (see (41) for the details), we generalized the main results in [27] to the stratified Lie groups instead of nilpotent Lie groups.

This paper is organized as follows. In Section 2, we collect some known facts about the auxiliary function $m(x, W)$. Section 3 gives some estimates of kernel for some operators in this paper. Section 4 gives the proof of the boundedness of $L^{-1} W, L^{-1}(-\Delta)$ on the space $B M O_{L}(G)$. In Section 5 , we establish the $B M O_{L}$ boundedness of $L^{-1} \nabla^{2}$. Finally, we give some examples for the potentials which satisfy the assumptions in Theorem 6 in different settings.

Throughout this paper, we will use $C$ to denote the positive constant, which is not necessarily the same at each occurrence and may depend on the dimension $Q$, and the constant in (9). By $A \sim B$ and $A \lesssim B$, we mean that there exist some constants $C, C^{\prime}$ such that $1 / C \leq A / B \leq C$ and $A \leq C^{\prime} B$, respectively.

\section{Some Lemmas about the Auxiliary Function}

In this section, we collect some known results about auxiliary function $m(x, W)$. We refer to [13] for the details. Throughout this section, unless otherwise indicated, we always assume that 0 三 $W \in B_{s}$ for some $s>Q / 2$.

Lemma 7. $W \in B_{s}$ is a doubling measure; that is, there exists a constant $C>0$ such that

$$
\int_{B(x, 2 r)} W(y) d y \leq C \int_{B(x, r)} W(y) d y .
$$

Lemma 8. There exist constants $C, k_{0}>0$ such that

$$
\begin{aligned}
\frac{1}{C}(1 & +d(x, y) m(x, W))^{-k_{0}} \\
& \leq \frac{m(x, W)}{m(y, W)} \\
& \leq C(1+d(x, y) m(x, W))^{k_{0} /\left(k_{0}+1\right)} .
\end{aligned}
$$

In particular, $m(x, W) \sim m(y, W)$ if $d(x, y) \leq C / m(x, W)$.

Lemma 9. There exists $C>0$ such that, for $0<r<R<\infty$,

$$
\begin{aligned}
& \frac{1}{r^{\mathrm{Q}-2}} \int_{B(x, r)} W(y) d y \\
& \quad \leq C\left(\frac{r}{R}\right)^{2-(\mathrm{Q} / s)} \frac{1}{R^{\mathrm{Q}-2}} \int_{B(x, R)} W(y) d y .
\end{aligned}
$$

Lemma 10. If $r=1 / m(x, W)$, then

$$
\frac{1}{r^{\mathrm{Q}-2}} \int_{B(x, r)} W(y) d y=1 \text {. }
$$

Moreover,

$$
\frac{1}{r^{\mathrm{Q}-2}} \int_{B(x, r)} W(y) d y \sim 1 \quad \text { iff } r \sim \frac{1}{m(x, W)} .
$$

Lemma 11. There exist $C>0$ and $l_{1}>0$ such that

$$
\begin{aligned}
& \int_{B(x, r)} \frac{W(y)}{d(x, y)^{\mathrm{Q}-2}} d y \\
& \quad \leq \frac{C}{r^{\mathrm{Q}-2}} \int_{B(x, r)} W(y) d y \leq C(1+r m(x, W))^{l_{1}} .
\end{aligned}
$$

Moreover, if $W \in B_{Q}$, then there exists $C>0$ such that

$$
\int_{B(x, r)} \frac{W(y) d y}{d(x, y)^{\mathrm{Q}-1}} \leq C \frac{1}{r^{\mathrm{Q}-1}} \int_{B(x, r)} W(y) d y .
$$




\section{Estimates for the Kernels}

In this section we will investigate some necessary estimates about the kernel of the operators in the paper.

Let $p_{t}(x, y)=h_{t}\left(y^{-1} x\right)$ be the heat kernel of the semigroup $e^{-t \Delta}, t \geq 0$, associated with $-\Delta$. Via Theorem 4.2 of [31], the following estimates hold true; that is, there exist positive constants $C$ and $C^{\prime}$ such that

$$
\begin{array}{r}
\left|\nabla^{2} h_{t}(x)\right| \leq C t^{-1} V(\sqrt{t})^{-1} \exp \left\{-C^{\prime} \frac{d(e, x)}{t}\right\}, \\
\forall x \in G, \forall t>0, \\
\left|\nabla^{3} h_{t}(x)\right| \leq C t^{-3 / 2} V(\sqrt{t})^{-1} \exp \left\{-C^{\prime} \frac{d(e, x)}{t}\right\}, \\
\forall x \in G, \forall t>0,
\end{array}
$$

where $e$ is the unit element of $G$. Moreover, for any $1 \leq i, j, k \leq$ $m$ and $x, y \in G$, by using (3.5) in [13] we obtain

$$
\begin{aligned}
& \left|X_{i}^{(x)} X_{j}^{(x)} X_{k}^{(y)} p_{t}(x, y)\right| \\
& \quad \leq C t^{-3 / 2} V^{-1}(\sqrt{t}) \exp \left\{-C^{\prime} \frac{d^{2}(x, y)}{t}\right\} .
\end{aligned}
$$

Let

$$
H_{t} f(x) \doteq \int_{G} f(y) h_{t}\left(y^{-1} x\right) d y, \quad \forall t>0 .
$$

Then for $\lambda \geq 0$,

$$
(\lambda-\Delta)^{-1} f(x)=\int_{0}^{\infty} e^{-\lambda t} H_{t} f(x) d t .
$$

Let $\Gamma_{0}(x, y, \lambda)$ be the fundamental solution of the operator $-\Delta+\lambda$ for $\lambda \in[0, \infty)$. In particular, we denote by $\Gamma_{0}(x, y, 0)=$ $\Gamma_{0}(x, y)$. Then we have the following.

Proposition 12. There exists a positive constant $C_{k}$ such that

$$
\begin{aligned}
\left|\Gamma_{0}(x, y, \lambda)\right| & \leq \frac{C_{k}}{\left(1+\lambda^{1 / 2} d(x, y)\right)^{k}} \frac{1}{d^{\mathrm{Q}-2}(x, y)}, \\
\left|\nabla_{x} \Gamma_{0}(x, y, \lambda)\right| & \leq \frac{C_{k}}{\left(1+\lambda^{1 / 2} d(x, y)\right)^{k}} \frac{1}{d^{\mathrm{Q}-1}(x, y)}, \\
\left|\nabla_{x}^{2} \Gamma_{0}(x, y, \lambda)\right| & \leq \frac{C_{k}}{\left(1+\lambda^{1 / 2} d(x, y)\right)^{k}} \frac{1}{d^{\mathrm{Q}}(x, y)}, \\
\left|\nabla_{x}^{2} \nabla_{y} \Gamma_{0}(x, y, \lambda)\right| & \leq \frac{C_{k}}{\left(1+\lambda^{1 / 2} d(x, y)\right)^{k}} \frac{1}{d^{\mathrm{Q}+1}(x, y)}, \\
\left|\nabla_{x}^{3} \Gamma_{0}(x, y, \lambda)\right| & \leq \frac{C_{k}}{\left(1+\lambda^{1 / 2} d(x, y)\right)^{k}} \frac{1}{d^{\mathrm{Q}+2}(x, y)}
\end{aligned}
$$

for $x \neq y$.
Proof. Equations (29) and (30) have been proved by Li in [13]. We only need to show that (32) holds true, because (31) and (33) can be proved similarly.

By (26) and (28),

$$
\begin{aligned}
& \left|X_{i}^{2} X_{j}^{y} \Gamma_{0}(x, y, \lambda)\right| \\
& =\left|\int_{0}^{\infty} e^{-\lambda t} X_{i}^{2} X_{j}^{y} p_{t}(x, y) d t\right| \\
& \leq\left|\int_{0}^{d^{2}(x, y)} e^{-\lambda t} X_{i}^{2} X_{j}^{y} p_{t}(x, y) d t\right| \\
& \quad+\left|\int_{d^{2}(x, y)}^{\infty} e^{-\lambda t} X_{i}^{2} X_{j}^{y} p_{t}(x, y) d t\right| \doteq I_{1}+I_{2} .
\end{aligned}
$$

Firstly, for $I_{1}$, we have

$$
\begin{aligned}
I_{1} & \leq C\left|\int_{0}^{d^{2}(x, y)} e^{-\lambda t} t^{-3 / 2} V^{-1}(\sqrt{t}) \exp \left\{-C^{\prime} \frac{d^{2}(x, y)}{t}\right\} d t\right| \\
& \leq C\left|\int_{0}^{d^{2}(x, y)} e^{-\lambda t} t^{-3 / 2}\left(\frac{1}{\sqrt{t}}\right)^{\mathrm{Q}} \exp \left\{-C^{\prime} \frac{d^{2}(x, y)}{t}\right\} d t\right| \\
& \leq C\left|\int_{0}^{d^{2}(x, y)} e^{-\lambda t} t^{-3 / 2}\left(\frac{1}{\sqrt{t}}\right)^{\mathrm{Q}}\left(\frac{\sqrt{t}}{d(x, y)}\right)^{\mathrm{Q}+2} d t\right| \\
& \leq \frac{C}{d^{\mathrm{Q}+1}(x, y)} .
\end{aligned}
$$

In addition, for any positive integer $k$,

$$
\begin{aligned}
& \leq C\left|\int_{0}^{I^{2}(x, y)} e^{-\lambda t} t^{-3 / 2} V^{-1}(\sqrt{t}) \exp \left\{-C^{\prime} \frac{d^{2}(x, y)}{t}\right\} d t\right| \\
& \leq C\left|\int_{0}^{d^{2}(x, y)} e^{-\lambda t} t^{-3 / 2}\left(\frac{1}{\sqrt{t}}\right)^{\mathrm{Q}} \exp \left\{-C^{\prime} \frac{d^{2}(x, y)}{t}\right\} d t\right| \\
& \leq C\left|\int_{0}^{d^{2}(x, y)} e^{-\lambda t} t^{-3 / 2}\left(\frac{1}{\sqrt{t}}\right)^{\mathrm{Q}}\left(\frac{\sqrt{t}}{d(x, y)}\right)^{\mathrm{Q}+k+1} d t\right| \\
& \leq C\left|\int_{0}^{d^{2}(x, y)} e^{-\lambda t} t^{(k / 2)-1} d t\right| \frac{1}{d^{\mathrm{Q}+k+1}(x, y)} \\
& =C\left|\int_{0}^{\lambda d^{2}(x, y)} e^{-t} t^{(k / 2)-1} d t\right| \frac{1}{\left(\lambda^{1 / 2} d(x, y)\right)^{k} d^{\mathrm{Q}+1}(x, y)} \\
& \leq \frac{C_{k}}{d^{\mathrm{Q}+1}(x, y)\left(\lambda^{1 / 2} d(x, y)\right)^{k}} .
\end{aligned}
$$

Therefore,

$$
I_{1} \leq \frac{C_{k}}{\left(1+\lambda^{1 / 2} d(x, y)\right)^{k}} \frac{1}{d^{\mathrm{Q}+1}(x, y)} .
$$


Secondly, we have

$$
\begin{aligned}
& \leq C\left|\int_{d^{2}(x, y)}^{I_{2}} e^{-\lambda t} t^{-3 / 2} V^{-1}(\sqrt{t}) \exp \left\{-C^{\prime} \frac{d^{2}(x, y)}{t}\right\} d t\right| \\
& \leq C\left|\int_{d^{2}(x, y)}^{\infty} e^{-\lambda t} t^{-3 / 2} d t\right| V^{-1}(d(x, y)) \\
& \leq C C_{k}\left|\int_{d^{2}(x, y)}^{\infty} t^{-3 / 2} \frac{1}{\left(1+\lambda^{1 / 2} t^{1 / 2}\right)^{k}} d t\right| V^{-1}(d(x, y)) \\
& \leq C C_{k}\left|\int_{d^{2}(x, y)}^{\infty} t^{-3 / 2} d t\right| \frac{1}{\left(1+\lambda^{1 / 2} d(x, y)\right)^{k}} V^{-1}(d(x, y)) \\
& \leq C C_{k} \frac{1}{\left(1+\lambda^{1 / 2} d(x, y)\right)^{k}} \frac{1}{d^{\mathrm{Q}+1}(x, y)} .
\end{aligned}
$$

Therefore, (32) holds true.

Moreover, we need some other basic facts of fundamental solutions for sub-Laplacian on the stratified Lie group $G$ (see [32]).

In the first place, we use the standard notations $\mathscr{D}, \mathscr{E}$, and $\mathscr{D}^{\prime}$ for the spaces of $C^{\infty}$ functions with compact support, $C^{\infty}$ functions, and distributions on $\mathscr{D}$.

A measurable function $f$ on $G$ will be called homogeneous of degree $\lambda(\lambda \in \mathbb{C})$ if $f \circ \delta_{r}=r^{\lambda} f$ for all $r>0$. Likewise, a distribution $\tau \in \mathscr{D}^{\prime}$ will be called homogeneous of degree $\lambda$ if $\left\langle\tau, u \circ \delta_{r}\right\rangle=r^{-Q-\lambda}\langle\tau, u\rangle$ for all $u \in \mathscr{D}$ and $r>0$. A distribution which is $C^{\infty}$ away from $e$ and homogeneous of degree $\alpha-Q$ will be called a kernel of type $\alpha$.

A differential operator $D$ will be called homogeneous of degree $\lambda$ if $D\left(u \circ \delta_{r}\right)=r^{\lambda}(D u) \circ \delta_{r}$ for all $u \in \mathscr{D}$ and $r>0$. Since $G$ is stratified, $X \in \mathfrak{g}$ is homogeneous of degree $j$ if and only if $X \in V_{j}$. In particular, sub-Laplacian $\Delta$ is homogeneous of degree 2. It follows from [32] that if $K$ is a kernel of type $\alpha$ and $D$ is homogeneous of degree $\lambda$, then $D K$ is a kernel of type $\alpha-\lambda$.

For sub-Laplacian $\Delta, X_{i} X_{j} \Gamma_{0}$ is homogeneous of $-Q$ due to the fact that $\Gamma_{0}$ is homogeneous of $2-Q$, where $X_{i}, X_{j} \in V_{1}$. In addition, by using Proposition 1.7 in [28], we have

$$
\left|\Gamma_{0}(x y)-\Gamma_{0}(x)\right| \leq C \frac{|y|}{|x|^{\mathrm{Q}+1}} \text { whenever }|y| \leq \frac{|x|}{2} \text {. }
$$

By [28], the left-invariant fields $X \in V_{1}$ are formally skewadjoint; that is,

$$
\int_{G} X f(y) g(y) d y=-\int_{G} f(y) X g(y) d y .
$$

Moreover, $X$ interacts with convolution in the following way:

$$
X(f * g)=f * X g
$$

Let $\Gamma(x, y, \lambda)$ be the fundamental solution of $L+\lambda$ for $\lambda \epsilon$ $[0, \infty)$. Then, Theorem 3.6 in [13] implies that

$$
\begin{aligned}
|\Gamma(x, y, \lambda)| \leq & \frac{1}{\left(1+\lambda^{1 / 2} d(x, y)\right)^{k}} \\
& \times \frac{C_{k}}{(1+m(x, W) d(x, y))^{k}} \frac{1}{d^{Q-2}(x, y)} .
\end{aligned}
$$

In particular, $\Gamma(x, y)=\Gamma(x, y, 0)=\Gamma(y, x, 0)$ is the fundamental solution of Schrödinger operator $L=-\Delta+W$, which satisfies the following.

(i) For each $k \in \mathbb{N}$ there exists $C_{k}>0$ such that

$$
|\Gamma(x, y)| \leq \frac{C_{k}}{(1+m(x, W) d(x, y))^{k}} \frac{1}{d^{Q^{-2}}(x, y)} .
$$

(ii) If $W \in B_{\mathrm{Q}}$, then for each $k \in \mathbb{N}$ there exists $C_{k}>0$ such that

$$
|\nabla \Gamma(x, y)| \leq \frac{C_{k}}{(1+m(x, W) d(x, y))^{k}} \frac{1}{d^{Q-1}(x, y)},
$$

where the above estimate can be deduced by Lemma 5.1 in [13].

The operator $\nabla^{2}(-\Delta+W)^{-1}$ is defined by

$$
\nabla^{2}(-\Delta+W)^{-1} f(x)=\int_{G} K(x, y) f(y) d y
$$

where the kernel $K(x, y)=\nabla_{x}^{2} \Gamma(x, y)$. Also, its adjoint operator $(-\Delta+W)^{-1} \nabla^{2}$ is defined by

$$
(-\Delta+W)^{-1} \nabla^{2} f(x)=\int_{G} \widetilde{K}(x, y) f(y) d y
$$

where the kernel $\widetilde{K}(x, y)=\nabla_{y}^{2} \Gamma(x, y)$. Since $\Gamma(x, y)=\Gamma(y, x)$, then $\nabla_{y}^{2} \Gamma(x, y)=\nabla_{y}^{2} \Gamma(y, x)$.

Moreover, we also need other estimates for the kernel $K(x, y)$ and $\widetilde{K}(x, y)$ in order to prove the main results.

Lemma 13. Assume $W \in B_{s}$ for $s>Q$. Let $x_{0}, y_{0} \in G$ and $R=(1 / 2) d\left(x_{0}, y_{0}\right)$. Then for any $k \in \mathbb{N}$ there exists $C_{k}>0$ such that

$$
\left\|\nabla^{2} \Gamma\left(x, y_{0}\right)\right\|_{L^{s}\left(B\left(x_{0}, R\right)\right)} \leq \frac{C_{k} V(R)^{1 / s}}{\left(1+R m\left(x_{0}, W\right)\right)^{\left(k /\left(k_{0}+1\right)\right)-l_{1}}} \frac{1}{V(R)},
$$

where $k_{0}$ is the constant appearing in Lemma 8 and $l_{1}$ is the constant appearing in Lemma 11.

Proof. Let $u$ be the solution of $-\Delta+W=0$ in the ball $B\left(x_{0}, 2 R\right)$. By Lemma 3.2 in [13], we choose $\eta \in$ $C_{0}^{\infty}\left(B\left(x_{0}, 2 R\right)\right)$ such that $0 \leq \eta \leq 1, \eta=1$ on $B\left(x_{0}, R / C^{2}\right)$, $|\nabla \eta| \leq C^{*} / R$, and $\left|\nabla^{2} \eta\right| \leq C^{*} / R^{2}$, where $C \geq 1$ and $C^{*}$ are fixed constants, which are independent of $x_{0}$ and $R$. 
For $x \in B\left(x_{0}, R / 2 C^{2}\right)$,

$$
\begin{aligned}
u(x) \eta(x)= & \int_{G} \Gamma_{0}(x, z, \lambda)(-\Delta+\lambda)(u \eta)(z) d z \\
= & \int_{G} \Gamma_{0}(x, z, \lambda)(-W u \eta-2 \nabla u \cdot \nabla \eta-u \Delta \eta) d z \\
= & \int_{G} \Gamma_{0}(x, z, \lambda)(-W u \eta+u \Delta \eta) d z \\
& +2 \int_{G}\left[\nabla_{z} \Gamma_{0}(x, z, \lambda) \cdot \nabla \eta\right] u d z
\end{aligned}
$$

By (31) and (32) we have

$$
\begin{aligned}
& \left|\nabla_{x}^{2} u(x)\right| \\
& =\left|\int_{G} \nabla_{x}^{2} \Gamma_{0}(x, z, \lambda) W u \eta d z\right|+\left|\int_{G} \nabla_{x}^{2} \Gamma_{0}(x, z, \lambda) u \Delta \eta d z\right| \\
& \quad+2\left|\int_{G}\left[\nabla_{x}^{2} \nabla_{z} \Gamma_{0}(x, z, \lambda) \cdot \nabla \eta\right] u d z\right| \\
& \leq\left|\int_{G} \nabla_{x}^{2} \Gamma_{0}(x, z, \lambda) W u \eta d z\right|+\int_{G}\left|\nabla_{x}^{2} \Gamma_{0}(x, z, \lambda)\right||u \Delta \eta| d z \\
& \quad+2 \int_{G}\left|\nabla_{x}^{2} \nabla_{z} \Gamma_{0}(x, z, \lambda)\right||\nabla \eta||u| d z \\
& \leq\left|\int_{B\left(x_{0}, 2 R\right)} \nabla_{x}^{2} \Gamma_{0}(x, z, \lambda) W u \eta d z\right| \\
& \quad+2 \int_{B\left(x_{0}, 2 R\right)}\left|\nabla_{x}^{2} \nabla_{z} \Gamma_{0}(x, z, \lambda)\right||\nabla \eta||u| d z \\
& \quad\left|\int_{B\left(x_{0}, 2 R\right)} \nabla_{x}^{2} \Gamma_{0}(x, z, \lambda) W u \eta d z\right| \\
& \quad+\frac{C}{R^{2} V(R)} \int_{B\left(x_{0}, 2 R\right)}|u(z)| d z \\
& \leq \int_{B\left(x_{0}, 2 R\right)}\left|\nabla_{x}^{2} \Gamma_{0}(x, z, \lambda) W\right||u| d z \\
& \quad \int_{B\left(x_{0}, 2 R\right)}|u(z)| d z .
\end{aligned}
$$

By (9), the Calderón-Zygmund estimates, and Lemma 11,

$$
\begin{aligned}
& \left\|\nabla^{2} u\right\|_{L^{s}\left(B\left(x_{0}, R\right)\right)} \\
& \leq C\|W u \eta\|_{s}+C V(R)^{1 / s} R^{-2} \sup _{B\left(x_{0}, 2 R\right)}|u| \\
& \leq C V(R)^{1 / s} \\
& \quad \times\left\{\left(\frac{1}{V(R)} \int_{B\left(x_{0}, R\right)} W^{s}(z) d z\right)^{1 / s}+R^{-2}\right\} \sup _{B\left(x_{0}, 2 R\right)}|u|
\end{aligned}
$$

$$
\begin{aligned}
& \leq C V(R)^{1 / s}\left\{\left(\frac{1}{V(R)} \int_{B\left(x_{0}, R\right)} W(z) d z\right)+R^{-2}\right\} \sup _{B\left(x_{0}, 2 R\right)}|u| \\
& \leq C V(R)^{1 / s} R^{-2}\left\{\left(\frac{R^{2}}{V(R)} \int_{B\left(x_{0}, R\right)} W(z) d z\right)+1\right\} \sup _{B\left(x_{0}, 2 R\right)}|u| \\
& \leq C V(R)^{1 / s} R^{-2}\left(1+R m\left(x_{0}, W\right)\right)^{l_{1}} \cdot \sup _{B\left(x_{0}, 2 R\right)}|u| .
\end{aligned}
$$

Let $u(x)=\Gamma\left(x, y_{0}\right)$ and $R=(1 / 2) d\left(x_{0}, y_{0}\right)$. Then $u(x)=$ $\Gamma\left(x, y_{0}\right)$ is a solution of $-\Delta+W=0$ in $B\left(x_{0}, R\right)$. By the above inequality and Lemma 8 , we immediately have

$$
\begin{aligned}
& \left\|\nabla^{2} \Gamma\left(x, y_{0}\right)\right\|_{L^{s}\left(B\left(x_{0}, R\right)\right)} \\
& \quad \leq C V(R)^{1 / s} R^{-2}\left(1+R m\left(x_{0}, W\right)\right)^{l_{1}} \cdot \sup _{B\left(x_{0}, 2 r\right)}|u| \\
& \quad \leq \frac{C_{k} V(R)^{1 / s}}{\left(1+R m\left(x_{0}, W\right)\right)^{\left(k /\left(k_{0}+1\right)\right)-l_{1}}} \frac{1}{V(R)} .
\end{aligned}
$$

This finishes the proof of Lemma 13.

Lemma 14. Suppose $W \in B_{\text {s }}$ for some $s>Q$ and $|\nabla W| \in B_{s_{1}}$ for some $s_{1}>Q / 2$. Then

$$
\begin{aligned}
\left|\nabla^{2} \Gamma(x, y)-\nabla^{2} \Gamma_{0}(x, y)\right| & \\
\leq & \frac{C}{d^{\mathrm{Q}-2}(x, y)} \int_{B(x, d(x, y))} \frac{|\nabla W(z)| d z}{d^{\mathrm{Q}-1}(x, z)} \\
& +\frac{C}{d^{\mathrm{Q}-1}(x, y)}(d(x, y) m(y,|\nabla W|))^{\delta_{1}} \\
& +\frac{C}{d^{\mathrm{Q}}(x, y)}(d(x, y) m(y, W))^{\delta},
\end{aligned}
$$

where $\delta_{1}=2-Q / s_{1}$ and $\delta=2-Q / s$, if $d(x, y) \leq$ $A / m(x,|\nabla W|)$ for some positive constant $A$.

Proof. Note that $(-\Delta+W) \Gamma(x, y)=0$. Let $\Gamma_{0}(x, y)$ be the fundamental solution of $-\Delta$. Then, we have

$$
-\Delta\left(\Gamma(x, y)-\Gamma_{0}(x, y)\right)=-W(x) \Gamma(x, y) .
$$

It follows that

$$
\Gamma(x, y)-\Gamma_{0}(x, y)=(-\Delta)^{-1}(-W(x) \Gamma(x, y)) .
$$

By (41), we have

$$
\begin{aligned}
\nabla \Gamma & (x, y)-\nabla \Gamma_{0}(x, y) \\
& =-\int_{G} \nabla \Gamma_{0}(x, z) W(z) \Gamma(z, y) d z \\
& =-\int_{G} \Gamma_{0}(x, z) \nabla(W(z) \Gamma(z, y)) d z \\
& =-\int_{G} \Gamma_{0}(x, z)[\nabla W(z) \Gamma(z, y)+W(z) \nabla \Gamma(z, y)] d z .
\end{aligned}
$$


Set $R=(1 / 8) d(x, y)$. Thus, we have

$$
\begin{aligned}
& \left|\nabla^{2} \Gamma(x, y)-\nabla^{2} \Gamma_{0}(x, y)\right| \\
& \leq \int_{G}\left|\nabla \Gamma_{0}(x, z)\right||\nabla W(z)||\Gamma(z, y)| d z \\
& \quad+\int_{G}\left|\nabla \Gamma_{0}(x, z)\right||W(z)||\nabla \Gamma(z, y)| d z \\
& \quad \doteq I_{1}+I_{2} .
\end{aligned}
$$

For $I_{1}$,

$$
\begin{aligned}
I_{1} & \leq\left(\int_{d(x, z)<R}+\int_{d(y, z)<R}+\int_{d(x, z) \geq R, d(y, z) \geq R}\right) \\
& =I_{11}+I_{12}+I_{13} .
\end{aligned}
$$

Firstly, by (43) and (30), it holds that

$$
\begin{aligned}
I_{11} & =\int_{d(x, z)<R}\left|\nabla \Gamma_{0}(x, z)\right||\nabla W(z)||\Gamma(z, y)| d z \\
& \leq \frac{C}{d^{\mathrm{Q}-2}(x, y)} \int_{B(x, R)} \frac{|\nabla W(z)|}{d^{\mathrm{Q}-1}(x, z)} d z .
\end{aligned}
$$

Secondly, via Lemmas 9 and 11, we similarly have

$$
\begin{aligned}
I_{12} & =\int_{d(y, z)<R}\left|\nabla \Gamma_{0}(x, z)\right| \cdot|\nabla W(z)| \cdot|\Gamma(z, y)| d z \\
& \leq \frac{C}{d^{Q-1}(x, y)} \int_{B(y, R)} \frac{|\nabla W(z)|}{d^{\mathrm{Q}-2}(y, z)} d z \\
& \leq \frac{C}{d^{\mathrm{Q}-1}(x, y)} \frac{1}{d^{\mathrm{Q}-2}(x, y)} \int_{B(y, R)}|\nabla W(z)| d z \\
& \leq \frac{C}{d^{\mathrm{Q}-1}(x, y)}(d(x, y) m(x,|\nabla W|))^{\delta_{1}},
\end{aligned}
$$

where $\delta_{1}=2-\left(Q / s_{1}\right)$.

Now, we turn to estimating $I_{13}$. By Lemma 7, (43), and (30), we have that

$$
\begin{aligned}
I_{13}= & \int_{d(x, z) \geq R, d(y, z) \geq R}\left|\nabla \Gamma_{0}(x, z)\right| \cdot|\nabla W(z)| \cdot|\Gamma(z, y)| d z \\
\leq & \frac{C}{d^{Q-1}(x, y)} \\
& \times \sum_{j=1}^{\infty} \int_{2^{j-1} R \leq d(y, z)<2^{j} R}|\nabla W(z)| \\
\leq & \frac{C}{d^{Q-1}(x, y)} \times\left(d^{Q-2}(y, z)(1+d(y, z) m(y, W))^{k}\right)^{-1} d z \\
& \times \sum_{j=1}^{\infty} \frac{C^{j}}{2^{k j}} \frac{1}{\left(2^{j} c R\right)^{Q-2}} \int_{d(y, z) \leq R}|\nabla W(z)| d z
\end{aligned}
$$

$$
\begin{aligned}
\leq & \frac{C}{d^{\mathrm{Q}-1}(x, y)} \\
& \times \sum_{j=1}^{\infty}\left(\frac{C}{2^{k+n-2}}\right)^{j} \frac{1}{R^{\mathrm{Q}-2}} \int_{d(y, z) \leq R}|\nabla W(z)| d z \\
\leq & \frac{C}{d^{\mathrm{Q}-1}(x, y)}(d(x, y) m(y,|\nabla W|))^{\delta_{1}}
\end{aligned}
$$

if we choose $k$ large enough.

Then,

$$
\begin{aligned}
I_{1} \leq & \frac{C}{d^{\mathrm{Q}-2}(x, y)} \int_{B(x, R)} \frac{|\nabla W(z)|}{d^{\mathrm{Q}-1}(x, z)} d z \\
& +\frac{C}{d^{\mathrm{Q}-1}(x, y)}(d(x, y) m(y,|\nabla W|))^{\delta_{1}} .
\end{aligned}
$$

A similar argument implies that

$$
I_{2} \leq \frac{C}{d^{Q}(x, y)}\left(\frac{d(x, y)}{m(y, W)}\right)^{\delta} .
$$

The proof is completed.

Lemma 15. Suppose $W \in B_{s}$ for some $s \geq Q / 2$. Let $s^{\prime}$ be the conjugate index of $s$.

(1) $L^{-1}(-\Delta)$ and $L^{-1} W$ are bounded on the space $L^{p}(G)$, where $s^{\prime} \leq p \leq \infty$.

(2) $L^{-1} \nabla^{2}$ is bounded on the space $L^{p}(G)$ for $s^{\prime} \leq p<\infty$.

The above lemmas hold true due to Theorem 4.1 and Theorem A in [13], respectively.

\section{The Boundedness of $L^{-1} W$ and $L^{-1}(-\Delta)$ on $B M O_{L}(G)$}

Proof of Theorem 5. To prove Theorem 5, we adopt the method used in the proof of Theorem 1.6 in [27].

Suppose $f \in B M O_{L}(G)$ and $B=B\left(x_{0}, r\right)$. Firstly, we suppose $r \geq \rho\left(x_{0}\right)$. Set $B^{*}=B\left(x_{0}, 2 r\right)$. Then, we have

$$
f=f \chi_{B^{*}}+f \chi_{\left(B^{*}\right)^{c}}=f_{1}+f_{2},
$$

where $\chi_{A}$ denotes the characteristic function of the set $A$. Since $L^{-1} W$ is bounded on $L^{2}(G)$, by Remark 4 , we have

$$
\begin{aligned}
& \frac{1}{|B|} \int_{B}\left|\left(L^{-1} W\right) f_{1}(x)\right| d x \\
& \quad \leq\left(\frac{1}{|B|} \int_{B}\left|\left(L^{-1} W\right) f_{1}(x)\right|^{2} d x\right)^{1 / 2} \\
& \quad \leq\left(\frac{C}{|B|} \int_{B^{*}}|f(x)|^{2} d x\right)^{1 / 2} \leq C\|f\|_{B M O_{L}} .
\end{aligned}
$$


Let $x \in B$. By Lemma $8,1 / m(x, W) \leq C r$. Set $T_{j}=$ $B\left(x_{0}, 2^{j} r\right)$. By using (43) and Lemma 11, we obtain

$$
\begin{aligned}
& \left|\left(L^{-1} W\right) f_{2}(x)\right| \\
\leq & \int_{\left(B^{*}\right)^{c}}|\Gamma(x, y)| W(y)|f(y)| d y \\
\leq & m\left(x_{0}, W\right)^{-k} \sum_{j=0}^{\infty} \frac{C_{k}}{\left(2^{j} r\right)^{Q-2+k}} \\
& \times \int_{T_{j+1} \backslash T_{j}} W(y)|f(y)| d y \\
\leq & m\left(x_{0}, W\right)^{-k} \\
& \times \sum_{j=0}^{\infty} \frac{C_{k}}{\left(2^{j} r\right)^{k-2}}\left(\frac{1}{\left|T_{j+1}\right|} \int_{T_{j+1}} W(y)^{s} d y\right)^{1 / s}\|f\|_{B M O_{L}} \\
\leq & C_{k} \sum_{j=0}^{\infty} 2^{-j\left(k-l_{1}\right)} \\
& \times\left(\frac{1}{r m\left(x_{0}, W\right)}\right)^{k-l_{1}}\|f\|_{B M O_{L}} \\
\leq & C\|f\|_{B M O_{L}},
\end{aligned}
$$

where we choose $k$ large enough. Thus

$$
\frac{1}{|B|} \int_{B}\left|\left(L^{-1} W\right) f_{2}(x)\right| d x \leq C\|f\|_{B M O_{L}} .
$$

The above argument also shows that $L^{-1} W$ is well defined on $B M O_{L}(G)$ without the ambiguity of an additive constant.

Suppose $r<\rho\left(x_{0}\right)$. Set $B^{\natural}=B\left(x_{0}, 2 \rho\left(x_{0}\right)\right)$. Then, we can write

$$
f=f \chi_{B^{\natural}}+f \chi_{\left(B^{\natural}\right)^{c}}=f_{1}+f_{2} .
$$

Via Lemma $8, m(x, W) \sim m\left(x_{0}, W\right)$ for any $x \in B$. Similar to (66), we have

$$
\frac{1}{|B|} \int_{B}\left|\left(L^{-1} W\right) f_{2}(x)\right| d x \leq C\|f\|_{B M O_{L}}
$$

Note that

$$
\begin{aligned}
|f(B, W)-f(2 B, W)| & \leq C|f(2 B, W)| \\
& \leq \frac{C}{|2 B|} \int_{2 B}|f(y)| d y, \\
\left|f\left(B^{\natural}, W\right)\right| & \leq\|f\|_{B M O_{L}} .
\end{aligned}
$$

Set $T_{j}=B\left(x, 2^{j} \rho\left(x_{0}\right)\right)\left(j \in \mathbb{Z}^{-} \cup\{0,1\}\right)$. By Hölder inequality and (9), we have, for any $x \in B$,

$$
\begin{aligned}
& \left|\left(L^{-1} W\right) f_{1}(x)\right| \\
& \leq \int_{B^{\natural}}|\Gamma(x, y)| W(y)|f(y)| d y \\
& \leq \sum_{j=-\infty}^{1} \frac{C_{k}}{\left(2^{j} \rho\left(x_{0}\right)\right)^{Q-2}} \int_{T_{j} \backslash T_{j+1}} W(y)|f(y)| d y \\
& \leq C \sum_{j=-\infty}^{1} \frac{1}{\left(2^{j} \rho\left(x_{0}\right)\right)^{-2}} \\
& \quad \times\left(\frac{1}{\left|T_{j+1}\right|} \int_{T_{j+1}} W(y)^{s} d y\right)^{1 / s}|j+1|\|f\|_{B M O_{L}} \\
& \leq C \sum_{j=-\infty}^{1} 2^{j \delta}|j+1|\|f\|_{B M O_{L}} \\
& \leq C\|f\|_{B M O_{L}}
\end{aligned}
$$

where $\delta=2-(Q / s)$. Then, we have

$$
\frac{1}{|B|} \int_{B}\left|\left(L^{-1} W\right) f_{1}(x)-f_{1}\left(B^{\natural}, W\right)\right| d x \leq C\|f\|_{B M O_{L}} \cdot
$$

Therefore, we prove that $\left(L^{-1} W\right) f \in B M O_{L}(G)$ and $\left\|\left(L^{-1} W\right) f\right\|_{B M O_{L}} \leq C\|f\|_{B M O_{L}}$, where $C$ is an absolute constant independent of $f$.

Since $L^{-1} W+L^{-1}(-\Delta)=I d$, as an immediate consequence, $L^{-1}(-\Delta)$ is a bounded operator on $B M O_{L}(G)$.

The proof is completed.

\section{The Boundedness of $L^{-1} \nabla^{2}$ on $B M O_{L}(G)$}

Proof of Theorem 6. Similar to the proof of Theorem 1.7 in [27], we show that Theorem 6 holds true.

Suppose $f \in B M O_{L}(G)$ and $B=B\left(x_{0}, r\right)$. Firstly, we suppose $r \geq \rho\left(x_{0}\right)$. Set $B^{*}=B\left(x_{0}, 2 r\right)$. Then, we decompose $f$ as

$$
f=f \chi_{B^{*}}+f \chi_{\left(B^{*}\right)^{c}}=f_{1}+f_{2} .
$$

Due to Lemma 15, we conclude that $\widetilde{R}^{L}$ is bounded on $L^{2}(G)$. By Remark 4, we have

$$
\begin{aligned}
\frac{1}{|B|} \int_{B}\left|\widetilde{R}^{L} f_{1}(x)\right| d x & \leq\left(\frac{1}{|B|} \int_{B}\left|\widetilde{R}^{L} f_{1}(x)\right|^{2} d x\right)^{1 / 2} \\
& \leq\left(\frac{C}{|B|} \int_{B^{*}}|f(x)|^{2} d x\right)^{1 / 2} \\
& \leq C\|f\|_{B M O_{L}}
\end{aligned}
$$


Let $x \in B$. By Lemma $8,1 / m(x, W) \leq C r$. Set $T_{j}=$ $B\left(x_{0}, 2^{j} r\right)$. Then, by Hölder inequality and Lemma 13 , we have

$$
\begin{aligned}
& \left|\widetilde{R}^{L} f_{2}(x)\right| \\
& \leq \int_{\left(B^{*}\right)^{c}}\left|\nabla^{2} \Gamma(y, x)\right||f(y)| d y \\
& =\sum_{j=1}^{\infty} \int_{T_{j+1} \backslash T_{j}}\left|\nabla^{2} \Gamma(y, x)\right||f(y)| d y \\
& \leq \sum_{j=1}^{\infty}\left(\int_{T_{j+1} \backslash T_{j}}\left|\nabla^{2} \Gamma(y, x)\right|^{s} d y\right)^{1 / s}\left(\int_{T_{j+1} \backslash T_{j}}|f(y)|^{s^{\prime}} d y\right)^{1 / s^{\prime}} \\
& \leq \sum_{j=1}^{\infty} \frac{C}{\left(1+2^{j}\right)^{\left(k /\left(k_{0}+1\right)\right)-l_{1}}}\left(\frac{1}{\left|T_{j}\right|} \int_{T_{j}}|f(y)|^{s^{\prime}} d y\right)^{1 / s^{\prime}} \\
& \leq C\|f\|_{B M \mathrm{O}_{L}},
\end{aligned}
$$

where we choose $k$ sufficiently large.

Thus

$$
\frac{1}{|B|} \int_{B}\left|\widetilde{R}^{L} f_{2}(x)\right| d x \leq C\|f\|_{B M O_{L}} .
$$

The above argument also shows that $\widetilde{R}^{L}$ are well defined on $B M O_{L}(G)$ without the ambiguity of an additive constant.

Suppose $r<\rho\left(x_{0}\right)$. Set $B^{\natural}=B\left(x_{0}, 2 \rho\left(x_{0}\right)\right)$. Then, we can write $f$ as follows:

$$
f=f \chi_{B^{\natural}}+f \chi_{\left(B^{\natural}\right)^{c}}=f_{1}+f_{2} .
$$

Note that $m(x, W) \sim m\left(x_{0}, W\right)$ for any $x \in B$. Similar to (66), we have

$$
\frac{1}{|B|} \int_{B}\left|\widetilde{R}^{L} f_{2}(x)\right| d x \leq C\|f\|_{B M O_{L}} .
$$

To complete the proof of the theorem, by Remark 4, we only need to prove that there exists a constant $C_{B}$ such that

$$
\frac{1}{|B|} \int_{B}\left|\widetilde{R}^{L} f_{1}(x)-C_{B}\right| d x \leq C\|f\|_{B M O_{L}} .
$$

The left side of (78) is bounded by

$$
\frac{1}{|B|} \int_{B}\left|\widetilde{R}^{L} f_{1}(x)-\widetilde{R} f_{1}(x)\right| d x+\frac{1}{|B|} \int_{B}\left|\widetilde{R} f_{1}(x)-C_{B}\right| d x,
$$

where $\widetilde{R}=(-\Delta)^{-1} \nabla^{2}$ is the dual operators of classical higher order Riesz transform $R=\nabla^{2}(-\Delta)^{-1}$. Let $x \in B$ and $B_{x, k}=$ $B\left(x, 2^{2-k} \rho\left(x_{0}\right)\right), k=0,1, \ldots$ Note that $m(x, W) \sim m\left(x_{0}, W\right)$. It is clear that $\left|f\left(B_{x, 0}\right)\right| \leq C\|f\|_{B M O_{L}}$,

$$
\left|f\left(B_{x, k}\right)-f\left(B_{x, k-1}\right)\right| \leq C\|f\|_{B M O}
$$

(cf. [28, Page 148]); therefore,

$$
\left|f\left(B_{x, k}\right)\right| \leq C(k+1)\|f\|_{B M O_{L}} .
$$

It follows that

$$
\int_{B_{x, k}}|f(x)| d x \leq C(k+1)\left|B_{x, k}\right|\|f\|_{B M O_{L}} .
$$

By Lemma 14, we get

$$
\begin{aligned}
& \left|\widetilde{R}^{L} f_{1}(x)-\widetilde{R} f_{1}(x)\right| \\
& \leq \int_{B^{\natural}}\left|\nabla_{y}^{2} \Gamma(y, x)-\nabla_{y}^{2} \Gamma_{0}(y, x)\right||f(y)| d y \\
& \leq \sum_{k=0}^{\infty} \int_{B_{x, k} \backslash B_{x, k+1}}\left|\nabla_{y}^{2} \Gamma(y, x)-\nabla_{y}^{2} \Gamma_{0}(y, x)\right||f(y)| d y \\
& \leq C \sum_{k=0}^{\infty} \int_{B_{x, k} \mid B_{x, k+1}}\left\{\frac{1}{d^{Q-2}(x, y)} \int_{B(x, d(x, y))} \frac{|\nabla W(z)| d z}{d^{Q-1}(x, y)}\right. \\
& \quad+\frac{1}{d^{Q-1}(x, y)}(d(x, y) m(y,|\nabla W|))^{\delta_{1}} \\
& \left.\quad+\frac{C}{d^{Q}(x, y)}(d(x, y) m(y, W))^{\delta}\right\} \\
& \times|f(y)| d y=I_{1}+I_{2}+I_{3} .
\end{aligned}
$$

Since $y \in B^{\natural}=B\left(x_{0}, 2 \rho\left(x_{0}\right)\right), m\left(x_{0}, W\right) \sim m(y, W)$. It is easy to see that

$$
\begin{aligned}
I_{3} & \leq C \sum_{k=0}^{\infty} 2^{-k \delta} \frac{1}{\left(2^{-k} \rho\left(x_{0}\right)\right)^{Q}} \int_{B_{x, k}}|f(y)| d y \\
& \leq C \sum_{k=0}^{\infty}(k+1) 2^{-k \delta}\|f\|_{B M O_{L}} \\
& \leq C\|f\|_{B M O_{L}} .
\end{aligned}
$$

By the same argument and noting that $M \leq m\left(x_{0},|\nabla W|\right) \leq$ $C m\left(x_{0}, W\right) \leq 1 / r$, that is, $r \leq \rho\left(x_{0}\right) \leq C \rho_{1}\left(x_{0}\right) \leq M$,

$$
\begin{aligned}
I_{2} & \leq C \sum_{k=0}^{\infty} 2^{-k\left(1+\delta_{1}\right)} \frac{1}{\left(2^{-k} \rho\left(x_{0}\right)\right)^{\mathrm{Q}}} \int_{B_{x, k}}|f(y)| d y \\
& \leq C \sum_{k=0}^{\infty}(k+1) 2^{-k\left(1+\delta_{1}\right)}\|f\|_{B M O_{L}} \\
& \leq C\|f\|_{B M O_{L}} .
\end{aligned}
$$

Because $|\nabla W| \in B_{s_{1}}$ for $s_{1} \geq Q / 2$, then $\delta_{1} \geq 0$. Thus, the last series converges. Using the fractional integral and the condition $\rho\left(x_{0}\right) \leq C \rho_{1}\left(x_{0}\right) \leq M$, we have

$$
\begin{aligned}
I_{1} \leq & C \sum_{k=0}^{\infty} \frac{\left(2^{-k} \rho\left(x_{0}\right)\right)^{2}}{\left(2^{-k} \rho\left(x_{0}\right)\right)^{Q}}\left(\int_{B_{x, k-1}}|\nabla W|^{s_{1}} d y\right)^{1 / s_{1}} \\
& \times\left(\int_{B_{x, k-1}}|f(y)|^{p^{\prime}} d y\right)^{1 / p^{\prime}}
\end{aligned}
$$




$$
\begin{aligned}
& \leq C \rho\left(x_{0}\right) \sum_{k=0}^{\infty}(k+1) 2^{-k\left(1+\delta_{1}\right)}\|f\|_{B M O_{L}} \\
& \leq C\|f\|_{B M O_{L}},
\end{aligned}
$$

where $(1 / p)+\left(1 / p^{\prime}\right)=1$ and $1 / p=\left(1 / s_{1}\right)-(1 / Q)$. Thus

$$
\frac{1}{|B|} \int_{B}\left|\widetilde{R}^{L} f_{1}(x)-\widetilde{R} f_{1}(x)\right| d x \leq C\|f\|_{B M O_{L}}
$$

It remains to show

$$
\frac{1}{|B|} \int_{B}\left|\widetilde{R} f_{1}(x)-C_{B}\right| d x \leq C\|f\|_{B M O_{L}}
$$

Let $B_{k}=B\left(x_{0}, 2^{1-k} \rho\left(x_{0}\right)\right), k=0,1, \ldots, k_{0}$, where $k_{0}$ satisfies $2^{-k_{0}-1} \rho\left(x_{0}\right) \leq r<2^{-k_{0}} \rho\left(x_{0}\right)$. Note that $\widetilde{R} f_{1}=\widetilde{R}\left(f_{1}-f\left(B_{k_{0}}\right)\right)$. Set

$$
\begin{aligned}
f_{1}-f\left(B_{k_{0}}\right)= & \left(f_{1}-f\left(B_{k_{0}}\right)\right) \chi_{B_{k_{0}}} \\
& +\left(f_{1}-f\left(B_{k_{0}}\right)\right) \chi_{B_{0} \backslash B_{k_{0}}}-f\left(B_{k_{0}}\right) \chi_{\left(B_{0}\right)^{c}} \\
= & f_{1,1}+f_{1,2}+f_{1,3} .
\end{aligned}
$$

Since $\widetilde{R}$ are bounded on $L^{2}(G)$, by Remark 4 , we have

$$
\begin{aligned}
\frac{1}{|B|} \int_{B}\left|\widetilde{R} f_{1,1}(x)\right| d x & \leq\left(\frac{1}{|B|} \int_{B}\left|\widetilde{R} f_{1,1}(x)\right|^{2} d x\right)^{1 / 2} \\
& \leq\left(\frac{C}{\left|B_{k_{0}}\right|} \int_{B_{k_{0}}}\left|f_{1}-f\left(B_{k_{0}}\right)\right|^{2} d x\right)^{1 / 2} \\
& \leq C\|f\|_{B M O_{L}} .
\end{aligned}
$$

Since $\nabla^{2} \Gamma_{0}$ is homogeneous of degree $-Q$, then by (39),

$$
\begin{aligned}
& \left|\nabla_{y}^{2} \Gamma_{0}(y, x)-\nabla_{y}^{2} \Gamma_{0}\left(y, x_{0}\right)\right| \\
& \quad \leq \frac{C d\left(x, x_{0}\right)}{d\left(y, x_{0}\right)^{\mathrm{Q}+1}} \text { for } d\left(x, x_{0}\right) \leq \frac{d\left(y, x_{0}\right)}{2} .
\end{aligned}
$$

Then, for $x \in B$, we have

$$
\begin{aligned}
& \left|\widetilde{R} f_{1,2}(x)-\widetilde{R} f_{1,2}\left(x_{0}\right)\right| \\
& \leq \sum_{k=0}^{k_{0}-1} \int_{B_{k} \backslash B_{k+1}}\left|\nabla_{y}^{2} \Gamma_{0}(y, x)-\nabla_{y}^{2} \Gamma_{0}\left(y, x_{0}\right)\right| \\
& \quad \times\left|f_{1}(y)-f\left(B_{k_{0}}\right)\right| d y
\end{aligned}
$$

$$
\begin{aligned}
\leq C \sum_{k=0}^{k_{0}-1} \frac{2^{k-k_{0}}}{\left|B_{k}\right|} \int_{B_{k}} & \left(\left|f(y)-f\left(B_{k}\right)\right|\right. \\
& \left.+\left|f\left(B_{k}\right)-f\left(B_{k_{0}}\right)\right|\right) d y
\end{aligned}
$$$$
\leq C \sum_{k=0}^{k_{0}-1}\left(k_{0}-k+1\right) 2^{k-k_{0}}\|f\|_{B M O}
$$$$
\leq C\|f\|_{B M O} \text {. }
$$

Thus

$$
\frac{1}{|B|} \int_{B}\left|\widetilde{R} f_{1,2}(x)-\widetilde{R} f_{1,2}\left(x_{0}\right)\right| d x \leq C\|f\|_{B M O_{L}} .
$$

For the third term, we have

$$
\begin{aligned}
& \left|\widetilde{R} f_{1,3}(x)-\widetilde{R} f_{1,3}\left(x_{0}\right)\right| \\
& \leq\left|f\left(B_{k_{0}}\right)\right| \int_{\left(B_{0}\right)^{c}}\left|\nabla^{2} \Gamma_{0}(y, x)-\nabla^{2} \Gamma_{0}\left(y, x_{0}\right)\right| d y \\
& \leq C\left(k_{0}+1\right) 2^{-k_{0}}\|f\|_{B M O_{L}} \leq C\|f\|_{B M O_{L}} .
\end{aligned}
$$

Then

$$
\frac{1}{|B|} \int_{B}\left|\widetilde{R} f_{1,3}(x)-\widetilde{R} f_{1,3}\left(x_{0}\right)\right| d x \leq C\|f\|_{B M O_{L}} .
$$

The proof of Theorem 6 is completed.

\section{Example}

In this section, we give some examples for the potentials which satisfy the assumption in Theorem 6.

Case 1. Assume $G=\mathbb{R}^{n}$. At this time, the homogeneous norm $|\cdot|$ on $G$ is defined as

$$
|x|=\left(x_{1}^{2}+\cdots+x_{n}^{2}\right)^{1 / 2}
$$

for any $x \in \mathbb{R}^{n}$.

Example 1. Let $W(x)=A+|x|=A+\left(x_{1}^{2}+\cdots+x_{n}^{2}\right)^{1 / 2}$, where $A$ is a positive constant.

Following from [33], we know that if $P(x)$ is a polynomial and $\alpha>0$, then $W(x)=|P(x)|^{\alpha}$ belongs to $B_{\infty}\left(\mathbb{R}^{n}\right)$. For $1<p<\infty$, it is easy to see $B_{\infty}\left(\mathbb{R}^{n}\right) \subseteq B_{p}\left(\mathbb{R}^{n}\right)$.

Therefore, $W(x)=A+|x|=A+\left(x_{1}^{2}+\cdots+x_{n}^{2}\right)^{1 / 2} \epsilon$ $B_{\infty}\left(\mathbb{R}^{n}\right)$.

If $W_{1}(x) \leq C_{1} W_{2}(x)$, then

$$
\begin{aligned}
& \frac{1}{m\left(x, W_{1}\right)} \doteq \sup _{r>0}\left\{r: \frac{1}{r^{n-2}} \int_{B(x, r)} W_{1}(y) d y \leq 1\right\} \\
& \geq \frac{C_{2}}{m\left(x, W_{2}\right)} \doteq \sup _{r>0}\left\{r: \frac{1}{r^{n-2}} \int_{B(x, r)} W_{2}(y) d y \leq 1\right\},
\end{aligned}
$$


where $C_{1}$ and $C_{2}$ are positive constants. Thus,

$$
\nabla W(x)=\left(\frac{x_{1}}{\left(x_{1}^{2}+\cdots+x_{n}^{2}\right)^{1 / 2}}, \ldots, \frac{x_{n}}{\left(x_{1}^{2}+\cdots+x_{n}^{2}\right)^{1 / 2}}\right) .
$$

Therefore, $|\nabla W(x)|=1$. Clearly, $|\nabla W(x)| \in B_{\infty}\left(\mathbb{R}^{n}\right)$. So $W(x) \geq A|\nabla W(x)|$. Therefore, $\rho(x) \leq B_{1} \rho_{1}(x)$ and $\rho_{1}(x)=M_{1}$, where $M_{1}$ and $B_{1}$ are positive constants. Then the potential $W(x)=A+|x|=A+\left(x_{1}^{2}+\cdots+x_{n}^{2}\right)^{1 / 2}$ satisfies the assumption of Theorem 6 .

Example 2. Let $W(x)=A+|x|^{3}=A+\left(x_{1}^{2}+\cdots+x_{n}^{2}\right)^{3 / 2}$, where $A$ is a positive constant. By the above argument, we conclude that $W \in B_{\infty}\left(\mathbb{R}^{n}\right)$.

Then

$$
\nabla W(x)
$$

$$
=\left(3\left(x_{1}^{2}+\cdots+x_{n}^{2}\right)^{1 / 2} x_{1}, \ldots, 3\left(x_{1}^{2}+\cdots+x_{n}^{2}\right)^{1 / 2} x_{n}\right) .
$$

Thus, $|\nabla W(x)|=3|x|^{2}=3\left(x_{1}^{2}+\cdots+x_{n}^{2}\right)$. Clearly, $|\nabla W(x)| \in B_{\infty}\left(\mathbb{R}^{n}\right)$. From [5], we know that $m(x,|\nabla W|) \sim$ $1+|x|$. Furthermore, $\rho(x) \leq B_{2} \rho_{1}(x)$ and $\rho_{1}(x) \leq M_{2}$, where $B_{2}$ and $M_{2}$ are positive constants. Then the potential $W(x)=$ $A+|x|=A+\left(x_{1}^{2}+\cdots+x_{n}^{2}\right)^{3 / 2}$ satisfies the assumption of Theorem 6.

Case 2. Assume that $G=\mathbb{U}^{n}$. $\mathbb{\boxplus}^{n}$ is an nonabelian stratified Lie group. To begin with, we recall some basic facts on the Heisenberg group, which are found in [34]. The Heisenberg group $\mathbb{\square}^{n}$ is a Lie group with the underlying manifold $\mathbb{R}^{2 n} \times \mathbb{R}$ and the multiplication

$$
(x, t)(y, s)=\left(x+y, t+s+2 \sum_{j=1}^{n}\left(x_{n+j} y_{j}-x_{j} y_{n+j}\right)\right) .
$$

A basis for the Lie algebra of left-invariant vector fields on $\mathbb{\boxplus}^{n}$ is given by

$$
\begin{array}{r}
X_{j}=\frac{\partial}{\partial x_{j}}+2 x_{n+j} \frac{\partial}{\partial t}, \quad X_{n+j}=\frac{\partial}{\partial x_{n+j}}-2 x_{j} \frac{\partial}{\partial t}, \\
j=1, \ldots, n, X_{2 n+1}=\frac{\partial}{\partial t} .
\end{array}
$$

The gradient $\nabla_{\mathbb{H}^{n}}$ is defined by

$$
\nabla_{\mathbb{H}^{n}}=\left(X_{1}, \ldots, X_{2 n}\right) .
$$

$Q=2 n+2$ is the homogeneous dimension of $\mathbb{a}^{n}$. We define a homogeneous norm function on $\mathbb{T}^{n}$ by

$$
|g|=\left(|x|^{2}+|t|^{2}\right)^{1 / 4}, \quad g=(x, t) \in \mathbb{U}^{n} .
$$

By the equivalence of two quasi-norm in the finite dimension quasi-normed linear space, we conclude that, for any polynomial $P(g)$ on $\mathbb{Q}^{n}$ and $\alpha>0, W(g)=|P(g)|^{\alpha}$ belongs to $B_{\infty}$. For $1<p<\infty$, we also have $B_{\infty}\left(\mathbb{I}^{n}\right) \subseteq$ $B_{p}\left(\mathbb{\square}^{n}\right)$.
Example 3. For any $g=\left(x_{1}, \ldots, x_{2 n}, t\right) \in \mathbb{U}^{n}$, let $W(g)=$ $A+\left(x_{1}^{2}+\cdots+x_{2 n}^{2}\right)^{1 / 2}$, where $A$ is a positive constant. Then $W(g) \in B_{\infty}\left(\mathbb{Q}^{n}\right)$. Then

$$
\nabla W(g)=\left(\frac{x_{1}}{\left(x_{1}^{2}+\cdots+x_{2 n}^{2}\right)^{1 / 2}}, \ldots, \frac{x_{2 n}}{\left(x_{1}^{2}+\cdots+x_{2 n}^{2}\right)^{1 / 2}}\right)
$$

and $|\nabla W(g)|=1$. Clearly, $|\nabla W(g)| \in B_{\infty}\left(\mathbb{Q}^{n}\right)$. So $W(g) \geq$ $A|\nabla W(g)|$. Therefore, $\rho(x) \leq B_{3} \rho_{1}(x)$ and $\rho_{1}(x)=M_{3}$, where $M_{3}$ and $B_{3}$ are positive constants. Then the potential $W(g)=$ $A+\left(x_{1}^{2}+\cdots+x_{2 n}^{2}\right)^{1 / 2}$ satisfies the assumption of Theorem 6 .

Case 3. Assume that $G$ is a group of Heisenberg type with Lie algebra $\mathfrak{g}=V_{1} \oplus V_{2}$. Denote by $\left\{X_{1}, \ldots, X_{l}\right\}$ and $\left\{Y_{1}, \ldots, Y_{k}\right\}$ two orthonormal basis of $V_{1}$ and $V_{2}$, respectively. The group of Heisenberg type $G$ is a Lie group of step two with the underlying manifold $\mathbb{R}^{l} \times \mathbb{R}^{k}$ and the multiplication

$$
(x, y)\left(x^{\prime}, y^{\prime}\right)=\left(x+x^{\prime}, y+y^{\prime}+S\left(x, x^{\prime}\right)\right),
$$

where $x, x^{\prime} \in \mathbb{R}^{l}, y, y^{\prime} \in \mathbb{R}^{k}$, and $S\left(x, x^{\prime}\right)$ is a skewsymmetric bilinear function from $\mathbb{R}^{l} \times \mathbb{R}^{k}$ to $\mathbb{R}^{k}$ with integer coefficients when expressed in the standard bases of $\mathbb{R}^{l}$ and $\mathbb{R}^{k}$.

By [35], we know that there exist real constants $c_{i j m}$ with $i, j=1, \ldots, l, m=1, \ldots, k$, such that

$$
X_{i}=\frac{\partial}{\partial x_{i}}+\sum_{m=1}^{l} \sum_{j=1}^{k} c_{i j m} x_{m} \frac{\partial}{\partial y_{j}}, \quad i=1, \ldots, l .
$$

The gradient $\nabla_{G}$ is defined by

$$
\nabla_{G}=\left(X_{1}, \ldots, X_{l}\right) .
$$

$Q=l+2 k$ is the homogeneous dimension of $G$. We define a homogeneous norm function on $G$ by

$$
|g|=\left(|x|^{4}+|y|^{2}\right)^{1 / 4}, \quad g=(x, y) \in G .
$$

By the equivalence of two quasi-norm in the finite dimension quasi-normed linear space, we also conclude that, for any polynomial $P(g)$ on $G$ and $\alpha>0, W(g)=|P(g)|^{\alpha}$ belongs to $B_{\infty}$. For $1<p<\infty$, we also have $B_{\infty}(G) \subseteq B_{p}(G)$.

Example 4. For any $g=(x, y) \in G$, let $W(g)=$ $A+\left(x_{1}^{2}+\cdots+x_{l}^{2}\right)^{1 / 2}$, where $A$ is a positive constant. Then $W(g) \in B_{\infty}(G)$. Then

$$
\nabla W(g)=\left(\frac{x_{1}}{\left(x_{1}^{2}+\cdots+x_{l}^{2}\right)^{1 / 2}}, \ldots, \frac{x_{l}}{\left(x_{1}^{2}+\cdots+x_{l}^{2}\right)^{1 / 2}}\right)
$$

and $|\nabla W(g)|=1$. Clearly, $|\nabla W(g)| \in B_{\infty}(G)$. So $W(g) \geq$ $A|\nabla W(g)|$. Therefore, $\rho(x) \leq B_{4} \rho_{1}(x)$ and $\rho_{1}(x)=M_{4}$, where $M_{4}$ and $B_{4}$ are positive constants. Then the potential $W(g)=A+\left(x_{1}^{2}+\cdots+x_{l}^{2}\right)^{1 / 2}$ satisfies the assumption of Theorem 6. 


\section{Conflict of Interests}

The authors declare that there is no conflict of interests regarding the publication of this paper.

\section{Acknowledgments}

The authors are grateful to Professor Jie Xiao and Professor Hongquan Li for their helpful advice on this paper. This paper is supported by Research Fund for the Doctoral Program of Higher Education of China under Grant no. 20113108120001, the Shanghai Leading Academic Discipline Project (J50101), the National Natural Science Foundation of China under grant no. 10901018, and the Fundamental Research Funds for the Central Universities and Program for New Century Excellent Talents in University.

\section{References}

[1] J. Dziubański and J. Zienkiewicz, "Hardy space $H^{1}$ associated to Schrödinger operator with potential satisfying reverse Hölder inequality," Revista Matemática Iberoamericana, vol. 15, no. 2, pp. 279-296, 1999.

[2] J. Dziubański, G. Garrigós, T. Martínez, J. L. Torrea, and J. Zienkiewicz, "BMO spaces related to Schrödinger operators with potentials satisfying a reverse Hölder inequality," Mathematische Zeitschrift, vol. 249, no. 2, pp. 329-356, 2005.

[3] C. Fefferman and E. M. Stein, " $H^{p}$ spaces of several variables," Acta Mathematica, vol. 129, no. 3-4, pp. 137-193, 1972.

[4] Z. W. Shen, " $L^{p}$ estimates for Schrödinger operators with certain potentials," Annales de l'Institut Fourier, vol. 45, no. 2, pp. 513546, 1995.

[5] J. P. Zhong, Harmornic analysis for some Schrödinger type operators [Ph.D. thesis], Princeton University, Princeton, NJ, USA, 1993.

[6] Y. Liu and J. Dong, "Some estimates of higher order Riesz transform related to Schrödinger type operators," Potential Analysis, vol. 32, no. 1, pp. 41-55, 2010.

[7] J. Cao, Y. Liu, and Da. Yang, "Hardy spaces $H_{\mathscr{L}}^{1}\left(\mathbb{R}^{n}\right)$ associated to Schrödinger type operators $(-\Delta)^{2}+V^{2}$," Houston Journal of Mathematics, vol. 36, no. 4, pp. 1067-1095, 2010.

[8] J. Cao and Da. Yang, "Hardy spaces $H_{\mathscr{L}}^{p}\left(\mathbb{R}^{n}\right)$ associated with operators satisfying $k$-Davies-Gaffney estimates," Science China. Mathematics, vol. 55, no. 7, pp. 1403-1440, 2012.

[9] R. Jiang and Da. Yang, "Orlicz-Hardy spaces associated with operators satisfying Davies-Gaffney estimates," Communications in Contemporary Mathematics, vol. 13, no. 2, pp. 331-373, 2011.

[10] R. Jiang, Da. Yang, and Y. Zhou, "Orlicz-Hardy spaces associated with operators," Science in China. Series A, vol. 52, no. 5, pp. 1042-1080, 2009.

[11] Da. Yang, Do. Yang, and Y. Zhou, "Endpoint properties of localized Riesz transforms and fractional integrals associated to Schrödinger operators," Potential Analysis, vol. 30, no. 3, pp. 271-300, 2009.

[12] Da. Yang and Do. Yang, "Characterizations of localized $B M O\left(\mathbb{R}^{n}\right)$ via commutators of localized Riesz transforms and fractional integrals associated to Schrödinger operators," Collectanea Mathematica, vol. 61, no. 1, pp. 65-79, 2010.
[13] H.-Q. Li, "Estimations $L^{p}$ des opérateurs de Schrödinger sur les groupes nilpotents," Journal of Functional Analysis, vol. 161, no. 1, pp. 152-218, 1999.

[14] C. C. Lin, H. Liu, and Y. Liu, "Hardy spaces associatedwith Schrödinger operators on the Heisenberg group," http://arxiv.org/abs/1106.4960.

[15] C.-C. Lin and H. Liu, " $B M O_{L}\left(\mathbb{M}^{n}\right)$ spaces and Carleson measures for Schrödinger operators," Advances in Mathematics, vol. 228, no. 3, pp. 1631-1688, 2011.

[16] Y. Liu, "The weighted estimates for the operators $V^{\alpha}\left(-\Delta_{G}+V\right)^{-\beta}$ and $V^{\alpha} \nabla_{G}\left(-\Delta_{G}+V\right)^{-\beta}$ on the stratified Lie group G," Journal of Mathematical Analysis and Applications, vol. 349, no. 1, pp. 235-244, 2009.

[17] Y. Liu, J. Huang, and Do. Xie, "Some estimates of Schrödinger type operators on the Heisenberg group," Archiv der Mathematik, vol. 94, no. 3, pp. 255-264, 2010.

[18] Y. Liu, " $L^{p}$ estimates for Schrödinger type operators on the Heisenberg group," Journal of the Korean Mathematical Society, vol. 47, no. 2, pp. 425-443, 2010.

[19] Y. Liu, J. Z. Huang, and J. F. Dong, "An estimate on the heat kernel of Schrödinger operators with non-negative potentials on nilpotent Lie groups and its applications," Forum Mathematicum. In press.

[20] R. M. Gong, J. Li, and L. Song, "Besov and Hardy spaces associated with the Schrödinger operator on the Heisenberg group," Journal of Geometric Analysis, 2012.

[21] R. Jiang, X. Jiang, and Da. Yang, "Maximal function characterizations of Hardy spaces associated with Schrödinger operators on nilpotent Lie groups," Revista Matemática Complutense, vol. 24, no. 1, pp. 251-275, 2011.

[22] Da. Yang, Do. Yang, and Y. Zhou, "Localized BMO and BLO spaces on RD-spaces and applications to Schrödinger operators," Communications on Pure and Applied Analysis, vol. 9, no. 3, pp. 779-812, 2010.

[23] Da. Yang, Do. Yang, and Y. Zhou, "Localized MorreyCampanato spaces on metric measure spaces and applications to Schrödinger operators," Nagoya Mathematical Journal, vol. 198, pp. 77-119, 2010.

[24] Da. Yang and Y. Zhou, "Localized Hardy spaces $H^{1}$ related to admissible functions on $\mathrm{RD}$-spaces and applications to Schrödinger operators," Transactions of the American Mathematical Society, vol. 363, no. 3, pp. 1197-1239, 2011.

[25] J. Cao, Da. Yang, and S. Yang, "Endpoint boundedness of Riesz transforms on Hardy spaces associated with operators," Revista Matemática Complutense, vol. 26, no. 1, pp. 99-114, 2013.

[26] J. F. Dong and H. P. Liu, "The $B M O_{L}$ space and Riesz transforms associated with Schrödinger operators," Acta Mathematica Sinica, English Series, vol. 26, no. 4, pp. 659-668, 2010.

[27] J. F. Dong and Y. Liu, "The higher order Riesz transform and BMO type space associated to Schrödinger operators," Mathematische Nachrichten, vol. 285, no. 4, pp. 486-496, 2012.

[28] G. B. Folland and E. M. Stein, Hardy Spaces on Homogeneous Groups, vol. 28 of Mathematical Notes, Princeton University Press, Princeton, NJ, USA, 1982.

[29] A. Nagel, E. M. Stein, and S. Wainger, "Balls and metrics defined by vector fields. I. Basic properties," Acta Mathematica, vol. 155, no. 1-2, pp. 103-147, 1985.

[30] L. Hörmander, "Hypoelliptic second order differential equations," Acta Mathematica, vol. 119, pp. 147-171, 1967.

[31] N. Th. Varopoulos, L. Saloff-Coste, and T. Coulhon, Analysis and Geometry on Groups, vol. 100 of Cambridge Tracts in 
Mathematics, Cambridge University Press, Cambridge, Mass, USA, 1992.

[32] G. B. Folland, "Subelliptic estimates and function spaces on nilpotent Lie groups," Arkiv för Matematik, vol. 13, no. 2, pp. 161207, 1975.

[33] C. L. Fefferman, "The uncertainty principle," Bulletin of the American Mathematical Society, vol. 9, no. 2, pp. 129-206, 1983.

[34] E. M. Stein, Harmonic Analysis: Real-Variable Methods, Orthogonality, and Oscillatory Integrals, vol. 43 of Princeton Mathematical Series, Princeton University Press, Princeton, NJ, USA, 1993.

[35] L. Capogna, N. Garofalo, and D. Nhieu, "Properties of harmonic measures in the Dirichlet problem for nilpotent Lie groups of Heisenberg type," American Journal of Mathematics, vol. 124, no. 2, pp. 273-306, 2002. 


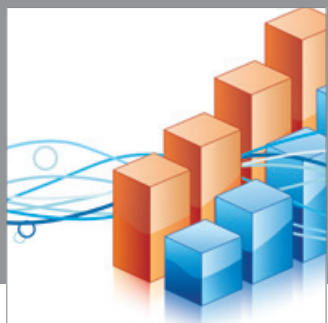

Advances in

Operations Research

mansans

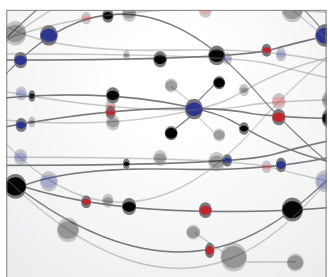

The Scientific World Journal
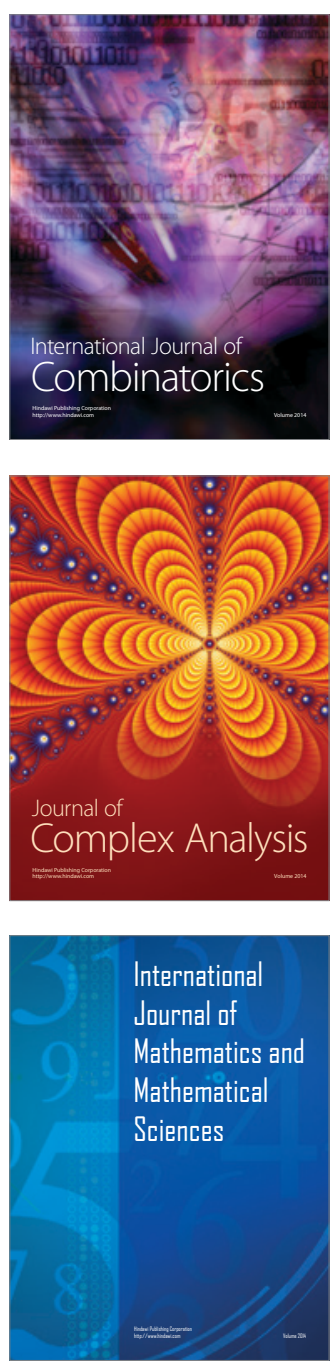
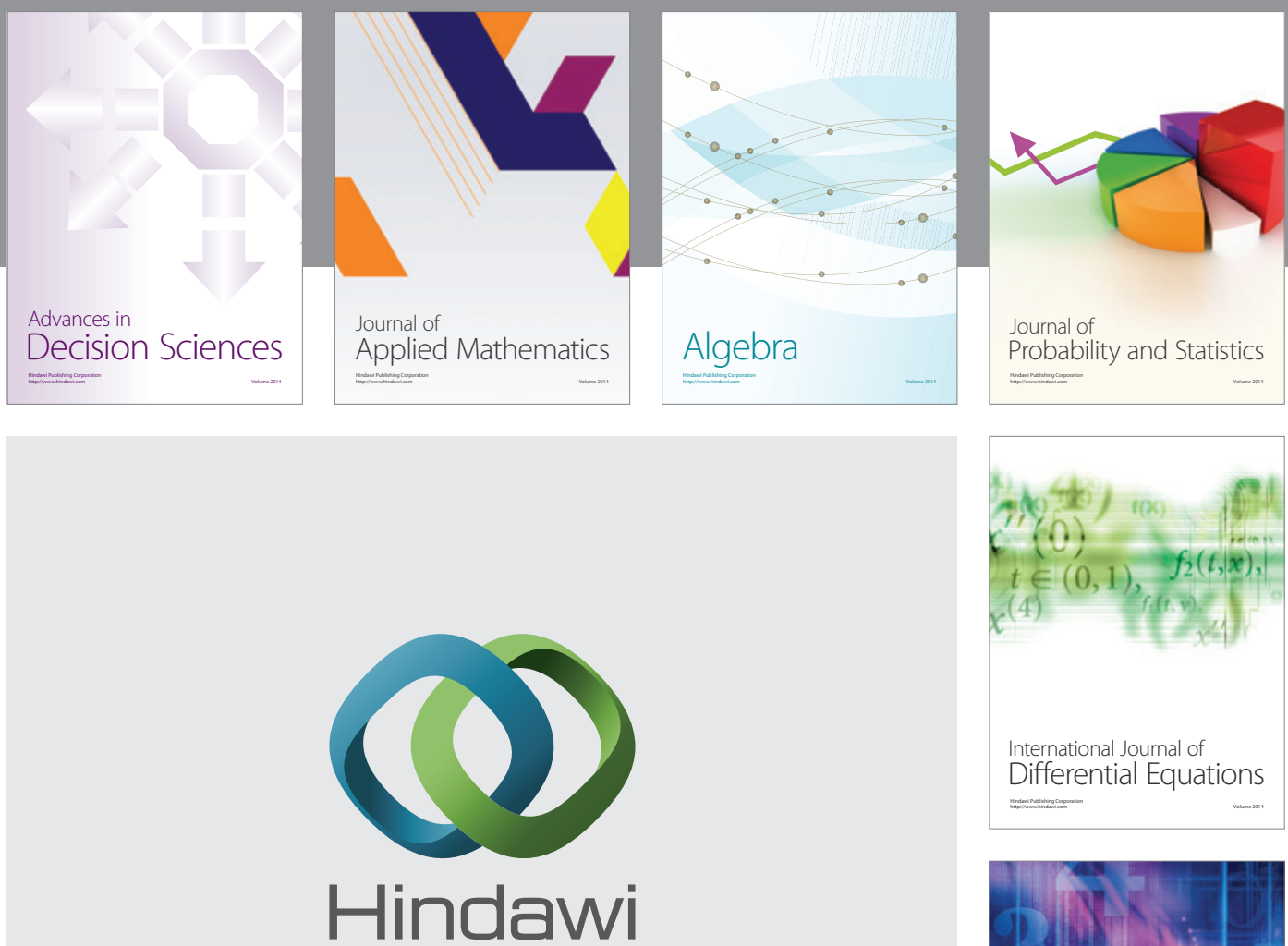

Submit your manuscripts at http://www.hindawi.com
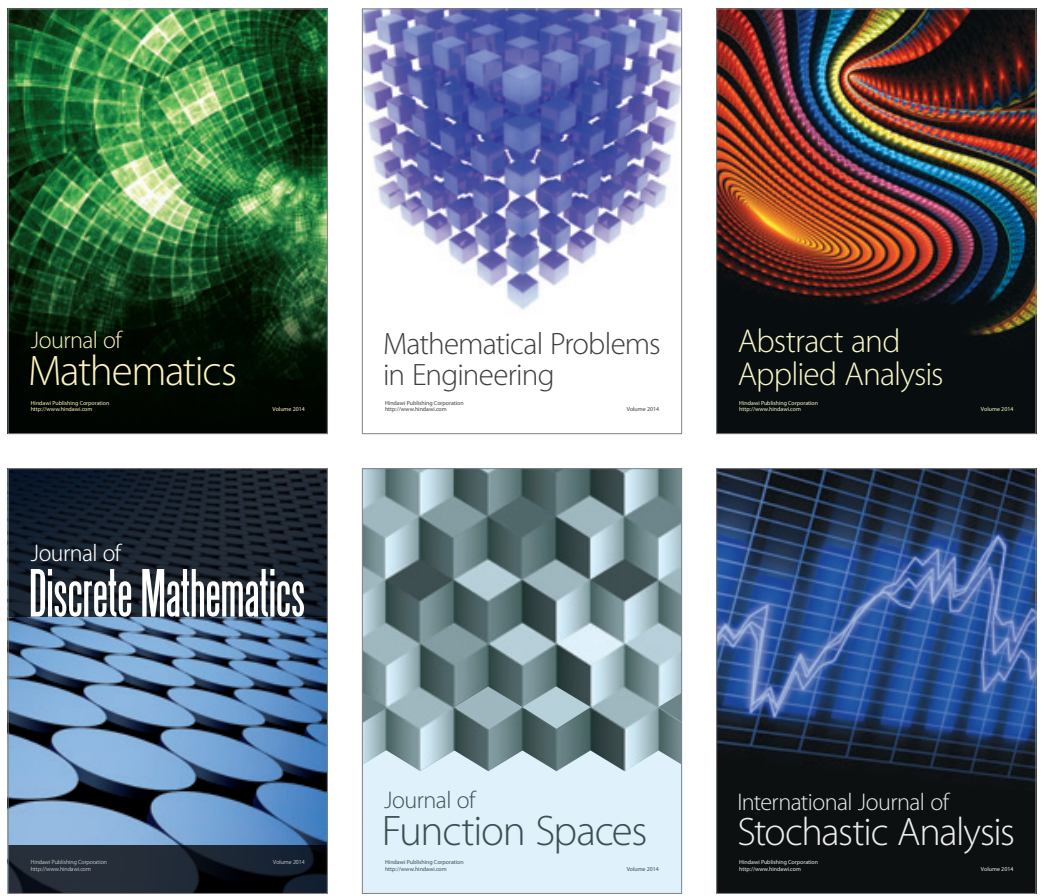

Journal of

Function Spaces

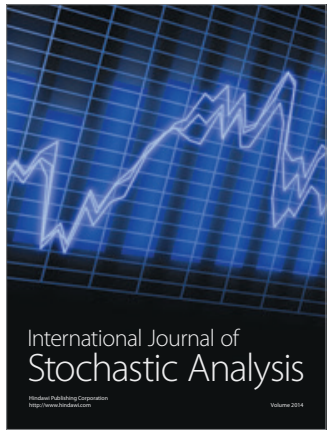

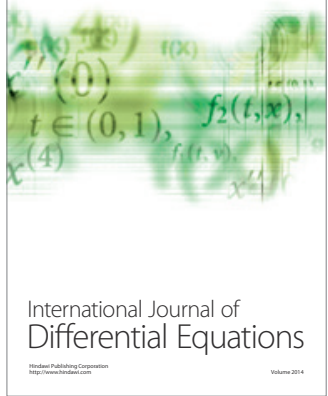
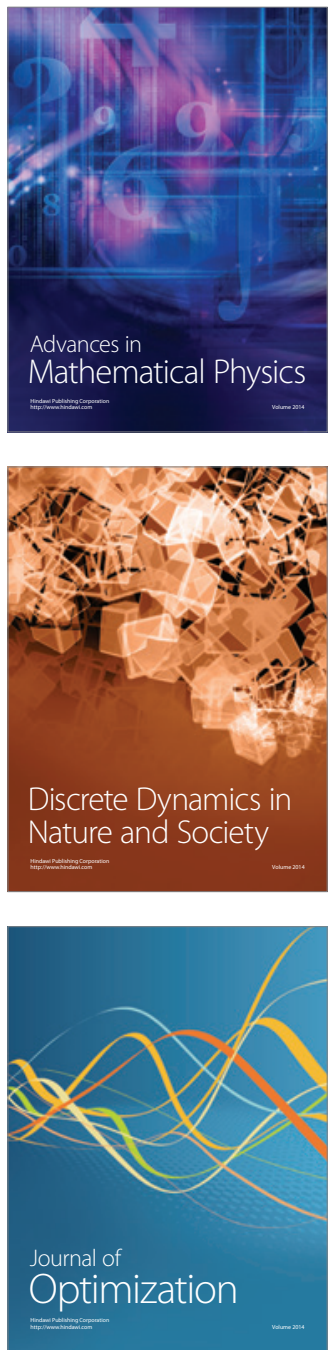